\title{
General practitioners' views of a psychogeriatric service
}

\author{
Olakunle Ashaye, Manu Dhadphale and Anthony Okore
}

\begin{abstract}
The views of referring general practitioners (GPs) on a psychogeriatic service were obtained through a questionnaire. Most GPs were satistied with the service. A number of GPs wanted faster responses to referrals, telephone discussions after intilal psychogeriatric assessments and more communication with consultants. In response to these findings, secretarial staff was increased and telephone discussions with referring GPs are to follow initial assessments of all urgent and emergency referrals.
\end{abstract}

Care in the community of the elderly mentally ill relies on good liaison between various agencies involved in their care. Prasher et al (1992) noted that the quality of care patients receive depends upon communication between general practitioners (GPs) and specialists.

There has been an increase in liaison schemes between GPs and old age psychiatrists (Barnerjee et al, 1993). Three main determinants of GP referrals to psychiatrists have been highlighted by Wilkinson (1989): GPs' characteristics; patients characteristics; and psychiatric services available.

The Barnet Healthcare NHS Trust provides services to residents of an outer London borough (Barnet) and part of a neighbourng shire county (Hertfordshire). The estimated number of persons over 65 years of age in the catchment area covered by the Trust is 56436 , based on the 1991 National Census figures. At the time of this study, the psychogeriatric service split the catchment area into two, with a consultant psychiatrist-led team for each sector. The services offered by both teams included in-patient care, day hospitals, outpatient clinics, crisis intervention and domiciliary visits for persons over 65 years of age.

Referrals to the services were categorised into emergency, urgent and routine. The emergency referrals were expected to be seen the same day with most assessments done by a crisis intervention team. This team offers a 24-hour service for emergency referrals to the adult and old age psychiatry (psychogeriatric) services. Members of the team include a junior doctor of registrar grade, a community psychiatric nurse and a social worker.
Urgent referrals were expected to be assessed within a week, and routine referrals within four weeks. The assessments were expected to be carried out by a psychogeriatric multidisciplinary team consisting of a psychiatrist, a social worker and a community psychiatric nurse. Collighan et al (1993) noted that multidisciplinary teamwork provides a means whereby valuable experiences of the several disciplines concerned can be applied to the widest possible range of those who need it. In view of the importance of good liaison between GPs and the psychogeriatric service, it was decided to find out the views of GPs on the responses to their referrals by the psychogeriatric multidisciplinary teams.

\section{The study}

All GPs who had referred patients between December 1993 and May 1994 to the psychogeriatric service were sent a questionnaire (Table 1). The audit advisory group for GPs in our catchment area was contacted before the questionnaires were sent out. They suggested amendments which were incorporated into the questionnaire. The questionnaire was kept as brief as possible to enhance the chances of response by GPs. Each questionnaire was sent with a stamped self-addressed envelope.

\section{Findings}

One hundred and twenty GPs made referrals to the service in the six month study period; 77 (64\%) responded and returned answered questionnaires. The responding GPs' estimated yearly referrals to the psychogeriatric service is shown in Table 2. Twenty-two (29\%) GPs said emergency referrals were seen on the same day. The rest either did not respond or said emergency referrals were seen within one and two weeks. Sixty-six (86\%) GPs said that they received a responding letter on assessment of their referrals. Seven $(9 \%)$ GPs said they received letters occasionally or rarely; four GPs gave no response.

Response letters on psychogeriatric multidisciplinary team assessments were received within a week of referral by $14(18 \%)$ GPs and in the 
Table 1. Questionnaire on GP referrals to the psychogeriatric service

1. How frequently do you refer patients to the psychogeriatric services yearly?

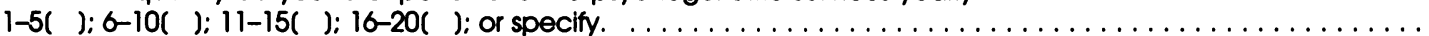

2. How quickly are your referrals responded to? Same day as referral( ); lst week( ); 2nd week( ); 3rd week( ) or

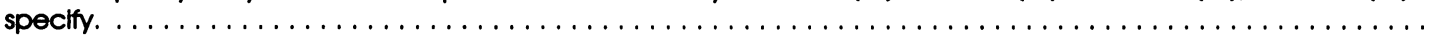

3. How frequently do you receive a letter of response following the assessment of the patient referred?

Never( ): Rarely( ): Occasionally( ): Most times( ): Always( ).

4. How quickly do you recelve the responding letter?

1st week( ): 2 nd week( ); 3 rd week( ); 4 th week( ); or specify. . . . . . . . . . . . . . . . . . . . . . . 5. Where would you like to see changes in our present response to your psychogeriatric referrals or to the senvice in

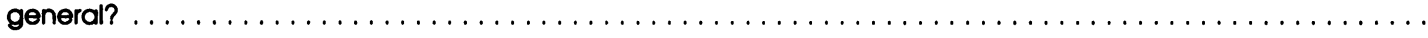

Table 2. Estimated yearly referrals to psychogeriatric service by 77 GPs

\begin{tabular}{ll}
$\begin{array}{l}\text { Estimated yearly } \\
\text { referrals }\end{array}$ & $\begin{array}{l}\text { Number of general } \\
\text { practitioners }\end{array}$ \\
\hline $1-5$ & $34(44 \%)$ \\
$6-10$ & $28(36 \%)$ \\
$11-15$ & $9(12 \%)$ \\
$16-20$ & $3(4 \%)$ \\
Not specified & $3(4 \%)$ \\
\hline
\end{tabular}

second week by 41 (53\%) GPs. Nine (12\%) GPs did not give a response and the rest gave estimations from three to over four weeks. Forty-nine (64\%) GPs said they were satisfied with present service and nine $(12 \%)$ wanted faster responses to referrals. Eight (10\%) GPs would have liked to have telephone discussions after initial multidisciplinary team assessments and $6(8 \%)$ wanted faster response letters following assessments. Other changes wanted by GPs are listed in Table 3.

\section{Comment}

This study reviewed GPs' opinions of a psychogeriatric service. It was encouraging that $77(64 \%)$ out of $120 \mathrm{GPs}$ responded to our questionnaire.

Table 3. Changes GPs would like to see in the psychogeriatric service

\begin{tabular}{ll}
\hline Response of GPs on changes wanted & $\begin{array}{l}\text { Number of } \\
\text { GPs }\end{array}$ \\
\hline Satisfied with service offered & $49(64 \%)$ \\
Faster response to referrals & $9(12 \%)$ \\
Telephone discussions after assessments & $8(10 \%)$ \\
Faster response letters & $6(8 \%)$ \\
More communication with the consultants & $5(6 \%)$ \\
Advice on medication & $3(4 \%)$ \\
Increased willingness to admit & 1 \\
Identification of individual key workers & 1 \\
Provision of incident records of follow-up & 1 \\
\hline
\end{tabular}

Most of the responders estimated they referred between one and ten patients yearly. Only 22 (29\%) GPs indicated that emergency referrals were seen on the day of referral. Thirty (39\%) GPs did not respond perhaps because they had made no emergency referrals, or because the emergency service is run with the adult psychiatric crisis service. Those GPs who felt responses were not done on the same day may have been unaware of the availability of this service when referring emergencies. As a result, an information sheet on the three types of psychogeriatric referrals has been produced and circulated to all GP surgeries in our catchment area (see Table 4).

The response time for urgent referrals has been reduced from one week to two working days. enabling the psychogeriatric multidisciplinary team to see their emergency and urgent referrals rather than the crisis intervention team.

The high number of GPs who said that they received response letters following assessments reflects the views of Prasher et al (1992) and Williams \& Wallace (1974) that the letter is the standard tool of communication between the GP and the specialist. Most GPs recetved responding letters to their referrals within two weeks. Identified reasons for delays included lack of secretarial cover and multidisciplinary team members' non-availability during vacations and sick leaves. Other reasons include inability to do initial assessments because referred patients are unavailable.

The secretarial staff has been increased by one secretary, enabling faster response letters to referring GPs. Most of the GPs who responded to our questionnaire were satisfied with the service offered. However, eight GPs wanted telephone discussions after the teams' initial assessments. It was decided that GPs would be contacted by phone after initial assessments of emergency or urgent referrals. Other commitments of team members, along with GPs' surgeries only being open at specified times make it difficult to give telephone responses to all referrals. 
Table 4. Information sheet to GPs on the psychogeriatric service

Following a questionnaire survey of referring general practtioners' views on the psychogeriatric service, we have produced this information sheet.

Our current practice with some of the changes to take place include:

1. Emergency referrals are to be seen on the day of referral by a psychogeriatric multidisciplinary team, or where this is not possible by a crisis intervention team. The latter team consists of a junior doctor of registrar grade, a social worker and a community psychiatric nurse. This team offers a 24 hours dally service for emergency referrals to both adult and old age psychiatric senvices. Referrals are made by telephone to the crisis intervention team's secretary.

2. Urgent referrals are to be seen within the next two working days of recelving referrals.

3. Initial assessments of both emergency and urgent referrals will be followed by telephone discussions with the referring GPS.

4. Response letters are to be sent to each referring GP within a week of initial assessment.

5. It is hoped in future to have regular meetings between various GP surgerles and the psychogeriatic service.

There are about 100 general practices in our catchment area. This large number made communication between all GPs and the two consultant psychiatrists for the elderly difficult. Banerjee et al (1993) noted the increasing liaison attachment schemes between psychiatrists and GPs. In our service, the multidisciplinary teams sometimes assess patients at GPs' surgeries, some of which have community psychiatric nurses.

In recognition of the need to improve liaison with GPs, the catchment area has been further divided into three sectors instead of two. Each sector is covered by a consultant psychiatrist along with other staff. It is expected that this will enable the psychogeriatric service in each sector to have regular meetings with GPs in their service.

There is a need for health services to adapt to the needs of the catchment population. This study gave us the opportunity to review our service in the light of GPs' comments and needs. identified in response to the questionnaires sent to them. As the primary health carers, GPs are in a position to give information on the health needs of the people they care for and services required.

\section{Acknowledgements}

We are grateful for the support we received from members of the psychogeriatric service. It was through the liaison of our audit department with the local medical audit advisory group for general practitioners that we were able to successfully carry out this study.

\section{References}

BARNERJEE, S., LINDESAY, J. \& MURPHY, E. (1993) Psychogeriatriclans and general practitioners: a national survey. Psychiatric Bulletin, 17, 592-594.

Colughan, G., Macdonald, A., HerzBerg, J., et al (1993) An evaluation of the multidisciplinary approach to psychiatric diagnoses in elderly people. British Medical Joumal, 306, 821-824.

Prasher, V. P., Fttzmaurice, D., Krishnan, V. H. R. et al (1992) Communication between general practitioners and psychiatrists. Psychiatric Bulletin. 16, 468-469

WiLkINSON, G. (1989) Referrals from general practitioners to psychiatrists and paramedical mental health professionals. British Journal of Psychiatry. 154, 72-76.

WInLAMS, P. \& WALAACE, B. B. (1974) General practitioners and psychiatrists - Do they communicate? British Medical Journal, 1, 505-507.

*Olakunle Ashaye, Registrar on the Charing Cross General Psychiatric Training Scheme; Manu Dhadphale, Consultant Psychiatrist for the Elderly, Barnet Psychiatric Unit, Barnet General Hospital, Wellhouse Lane, Barnet, Hertfordshire; Anthony Okore, Registrar on the Charing Cross General Psychiatric Training Scheme

*Correspondence: Dr. O. Ashaye, Cell Barnes Hospital, Highfield Lane, St. Albans, Hertfordshire ALA ORG 